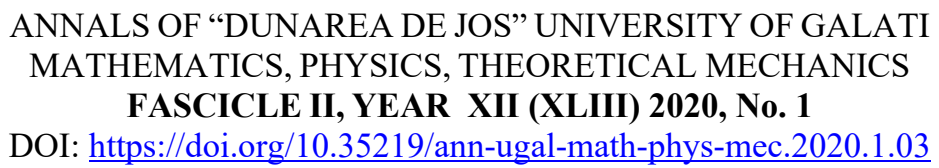

\title{
Comparison of distance metric measures to differentiate non-dermoscopic skin lesions
}

\author{
Felicia Anișoara Damian ${ }^{1}$, Simona Moldovanu $^{2,3}$, Luminița Moraru ${ }^{1 *}$ \\ ${ }^{1}$ Faculty of Sciences and Environment, “Dunarea de Jos” University of Galati, 47 Domneasca Street., RO- \\ 800008, Galati, Romania \\ ${ }^{2}$ Faculty of Automation, Computers Sciences, Electrical Engineering and Electronics, ”Dunarea de Jos” \\ University of Galati, 2 Stiintei Street, RO-800210, Galati, Romania \\ 3 "Dumitru Motoc" High School, 15 Milcov Street., RO-800509, Galati, Romania \\ * Corresponding author: luminita.moraru@ugal.ro
}

\begin{abstract}
The ABCD rule of dermoscopy is a way for a clinical diagnosis of melanoma that is based on low-level features such as asymmetry, border, color, and differential structures. They are currently used for solving various tasks related to the diagnosis of skin lesions. In this study, we are focusing on the diameter of the skin lesions (D criterion) as a tool to identify the most effective distance metric measures for the evaluation of pigmented lesions. The following distance measures were used: Euclidian and quasi-Euclidian, city block, chessboard distances. They were compared with a manual distance (as a ground truth data) for accurate results. We sorted 140 skin lesion images into two categories (nevi and melanomas) based on diameter and diagnosis. To validate and assess the clinical utility of the selected distance metrics, a receiver operating characteristic (ROC) curve and AUC (area under the curve) together with Youden were employed. Data from the ROC and AUC analysis suggest the chessboard distance as the sensitivity measure. It has higher values of sensitivity, specificity, and accuracy, for both nevi and melanoma. However, the diameter criterion (D) cannot be a stand-alone feature for melanoma detection but can be a valuable criterion and of increased suspicion for melanoma.
\end{abstract}

Keywords: distance metric measures, ROC curves, AUC

\section{INTRODUCTION}

Melanocytic nevus or mole is a benign skin lesion caused by the proliferation of pigmentproducing cells (melanocytes) [1]. Melanoma is a form of skin cancer with an aggressive evolution developed from a benign lesion and which is originated in the same pigment-producing cells [2]. Once this type of skin cancer is detected at an early stage, it becomes treatable.

Statistics published by the American Cancer Society which is based on information from the SEER database and the National Cancer Institute (NCI), show that early diagnosis of melanoma is crucial to improving survival (higher than $90 \%$ ).

Features based on size, shape, structure can be used to detect melanoma [3]. R.J. Friedman et al. [4] designed the $\mathrm{ABCD}$ criteria for melanoma screening to facilitate early diagnosis of cutaneous melanoma. These characteristics can be assessed according to the ABCDE clinical rule [5], the $\mathrm{ABCDEF}$ rule [6], the ABCD rule of dermatoscopy [7] or other methods. ABCDEF is an acronym for Asymmetry, irregular Border, at least three different Colors, Diameter, Evolution (i.e. significant change of the inspected lesion during time), and F denotes so called "ugly duck sign", i.e. a dissimilar appearance of malignant melanoma to the rest of melanocytic lesions. 
G. Grammatikopoulos et al. [8], proposed a total dermatoscopy score based on A, B, C, and D features. While A and D can be calculated with good accuracy, A and B suffer from a degree of inaccuracy. In order to calculate the diameter, the horizontal and vertical dimensions of the lesion were calculated as $\mathrm{h}=\max (\mathrm{i})-\min (\mathrm{i})$ and $\mathrm{v}=\max (\mathrm{j})-\min (\mathrm{j})$, where $(\mathrm{i}, \mathrm{j})$ denotes the coordinate of the pixels placed on the border of the lesion. The diameter is determined as $\mathrm{D}=\max (\mathrm{h}, \mathrm{v})$ in pixels, i.e. it would be the maximum of the two dimensions.

R. Garnavi et al. [9] proposed an original method to compute the greatest diameter and shortest diameter. The greatest diameter is defined as the distance between the farthest border points passing through the center of the lesion. The shortest diameter connects the two nearest boundary points and passes across the center of the skin lesion.

U. Kalwa et al. [10] proposed a smartphone application-based on the ABCD rule and a support vector machine (SVM) classifier to diagnose skin lesions as benign or malign. D is computed after an affine transformation (i.e. the lesion axes are aligned to the $\mathrm{x}-\mathrm{y}$ coordinate system) using the side length of the minimum area rectangle.

A.M. Glazer et al. [11] investigated the correlations between the quantitative $A B C D$ parameters and clinical observations of $\mathrm{ABCD}$. The values of $\mathrm{A}, \mathrm{B}, \mathrm{C}$, and $\mathrm{D}$ features were measured using a multispectral digital device. The diameters of studied lesions were from 2 to $22 \mathrm{~mm}$. The highest level of agreement (89\%) between quantitative and clinical observations of $\mathrm{ABCD}$ parameters was found in the diameter $\mathrm{D}$ feature.

N. Emiroglu et al. [12], determined the frequency of dermoscopic features in trunk melanomas based on the features such as the ratio of blue-gray veil, regression structures, milky-red areas, and multicomponent structures. Also, a statistical analysis according to the distribution of diameter values (less than $6 \mathrm{~mm}$, larger than $6 \mathrm{~mm}$ ) was performed by assessing dermoscopic and clinical images, and data provided by histopathological and clinical investigation. Their results indicated that dermoscopic features are statistically significant for large-diameter melanomas.

The present study aims to determine the performance of Euclidean (DE), chessboard (DC), city block (DB) and quasi-Euclidean (DQ) metrics in the estimation of the diameter feature values of the skin lesions, in digital images. The ground-truth is constructed by manual measurements. The performances of the DE, DC, DB, DQ measurements were analyzed using the ROC curves, the area under the curve (AUC) and the Youden Index (J), and related performance characteristics like sensitivity, specificity, and accuracy [13].

Afterward, the results of this study can be used for improving the apps devoted to skin lesion analysis.

\section{MATHEMATICAL APPROACHES}

a) Metric definition

A metric space $(X, d)$ is a set $X$ that has the concept of the distance $d(x, y)$ between any pair of points $\mathrm{x}, \mathrm{y} \in \mathrm{X}$. A metric is a function of the set $\mathrm{X}$, that satisfies the following properties for a distance [14]:

$$
\begin{aligned}
& \mathrm{d}(\mathrm{x}, \mathrm{y}) \geq 0 \forall \mathrm{x}, \mathrm{y} \varepsilon X,(\text { Non - negativity }) ; \\
& \mathrm{d}(\mathrm{x}, \mathrm{y})=0 \Leftrightarrow \mathrm{x}=\mathrm{y},(\text { Identity }) ; \\
& \mathrm{d}(\mathrm{x}, \mathrm{y})=\mathrm{d}(\mathrm{y}, \mathrm{x}) \forall \mathrm{x}, \mathrm{y} \varepsilon X,(\text { Symmetry }) ; \\
& \mathrm{d}(\mathrm{x}, \mathrm{y}) \leq \mathrm{d}(\mathrm{y}, \mathrm{z})+\mathrm{d}(\mathrm{z}, \mathrm{y}) \forall \mathrm{x}, \mathrm{y}, \mathrm{z} \varepsilon X,(\text { Triangular inequality) }
\end{aligned}
$$

b) Euclidean distance (DE)

In 2-D, $\mathrm{DE}$ between two points $\left(x_{1}, y_{1}\right)$ and $\left(x_{2}, y_{2}\right)$ is [15]:

$$
\mathrm{DE}=\sqrt{\left(\mathrm{x}_{1}-\mathrm{x}_{2}\right)^{2}+\left(\mathrm{y}_{1}-\mathrm{y}_{2}\right)^{2}}
$$

c) Chessboard distance (DC) 
In 2-D, DC between two points $\left(x_{1}, y_{1}\right)$ and $\left(x_{2}, y_{2}\right)$ is [16]:

$$
\mathrm{DC}=\max \left(\left|\mathrm{x}_{1}-\mathrm{x}_{2}\right|,\left|\mathrm{y}_{1}-\mathrm{y}_{2}\right|\right)
$$

d) City block distance (DB)

In 2-D, DB between two points $\left(x_{1}, y_{1}\right)$ and $\left(x_{2}, y_{2}\right)$ is [17]:

$$
\mathrm{DB}=\left|\mathrm{x}_{1}-\mathrm{x}_{2}\right|+\left|\mathrm{y}_{1}-\mathrm{y}_{2}\right|
$$

e) Quasi-Euclidean distance (DQ)

In 2-D, DB between two points $\left(x_{1}, y_{1}\right)$ and $\left(x_{2}, y_{2}\right)$ is [18]:

$$
\begin{aligned}
& \mathrm{DQ}=\left|\mathrm{x}_{1}-\mathrm{x}_{2}\right|+(\sqrt{2}-1)\left|\mathrm{y}_{1}-\mathrm{y}_{2}\right|, \text { for }\left|\mathrm{x}_{1}-\mathrm{x}_{2}\right|>\left|\mathrm{y}_{1}-\mathrm{y}_{2}\right| \\
& \mathrm{DQ}=(\sqrt{2}-1)\left|\mathrm{x}_{1}-\mathrm{x}_{2}\right|+\left|\mathrm{y}_{1}-\mathrm{y}_{2}\right|, \text { otherwise }
\end{aligned}
$$

\section{f) ROC curve (Receiver Operating Characteristics)}

The ROC curve is a two-dimensional curve displaying the sensitivity (probability of having a positive test when you are sick) on the Ox axis and (1-specificity) (false positive rate, i.e. the probability of having a negative test, when you are healthy) on the Oy axis. The following parameters are used: Sensitivity $=\mathrm{TP} /(\mathrm{TP}+\mathrm{FN})$ and Specificity $=\mathrm{TN} /(\mathrm{TN}+\mathrm{FP})$, respectively. $\mathrm{TP}$ is a positive prediction and positive response, $\mathrm{TN}$ is a negative prediction and negative response, $\mathrm{FP}$ is a positive prediction and negative response, and $\mathrm{FN}$ is a negative prediction and positive response. The ideal test is equally sensitive and specific [19].

The ROC curve provides a global assessment and the area under the curve (AUC) indicates how efficient a feature is, according to the declared goal [20]. The larger the AUC value, the more accurate the model is, and a better classification of the results is obtained [21]. Three basic elements of method validation are addressed: sensitivity (the proportion of a positive test among that cases knowing to have an issue), specificity (the proportion of a negative test among that cases not knowing to have an issue), and accuracy (the proportion of true results, either true positive or true negative).

g) Youden Index (J)

It is defined as $\mathrm{J}=$ maximum (Sensitivity + Specificity -1 ) and is another statistical interpretation of the ROC curve that allows the determination of the maximum cut-off point [22]. Lower J values indicate that the analyzed feature has no relevance for the study [23].

\section{METHOD}

The programming environment was MATLAB R2017a. The experimental hardware environment was a computer with Processor: Intel (R) Core (TM) i3-4030U, CPU 1.9 GHz, Installed memory (RAM): $4 \mathrm{~Gb}$, System type: 64-bit Operating System, x64-based processor. The ROC analysis was performed in the Medcalc application.

The dataset contains 140 color digital images (70 melanomas and 70 nevi), images from the digital archive of the Department of Dermatology within the University Medical Center of Groningen (UMCG), used for the development and testing of the MED-NODE system for skin cancer detection in RGB color digital images.

Proposed algorithm:

Input: RGB image

Output: distance measure values

\{Step 1\} Import RGB images;

\{Step 2$\}$ Convert RGB image to gray image; 
\{Step 3$\}$ Image binarization;

$\{$ Step 4$\}$ Measure the manual diameter of skin lesions;

\{Step 5 \} Compute DE, DC, DB and DQ metrics for diameter of skin lesions assessment;

\{Step 6\} Repeat steps 1-5 for 140 digital images containing skin lesions.

\{Step 7\} Plot the ROC curves and compute AUC and J performance indices;

Figure 1 shows the flowchart of the proposed method.

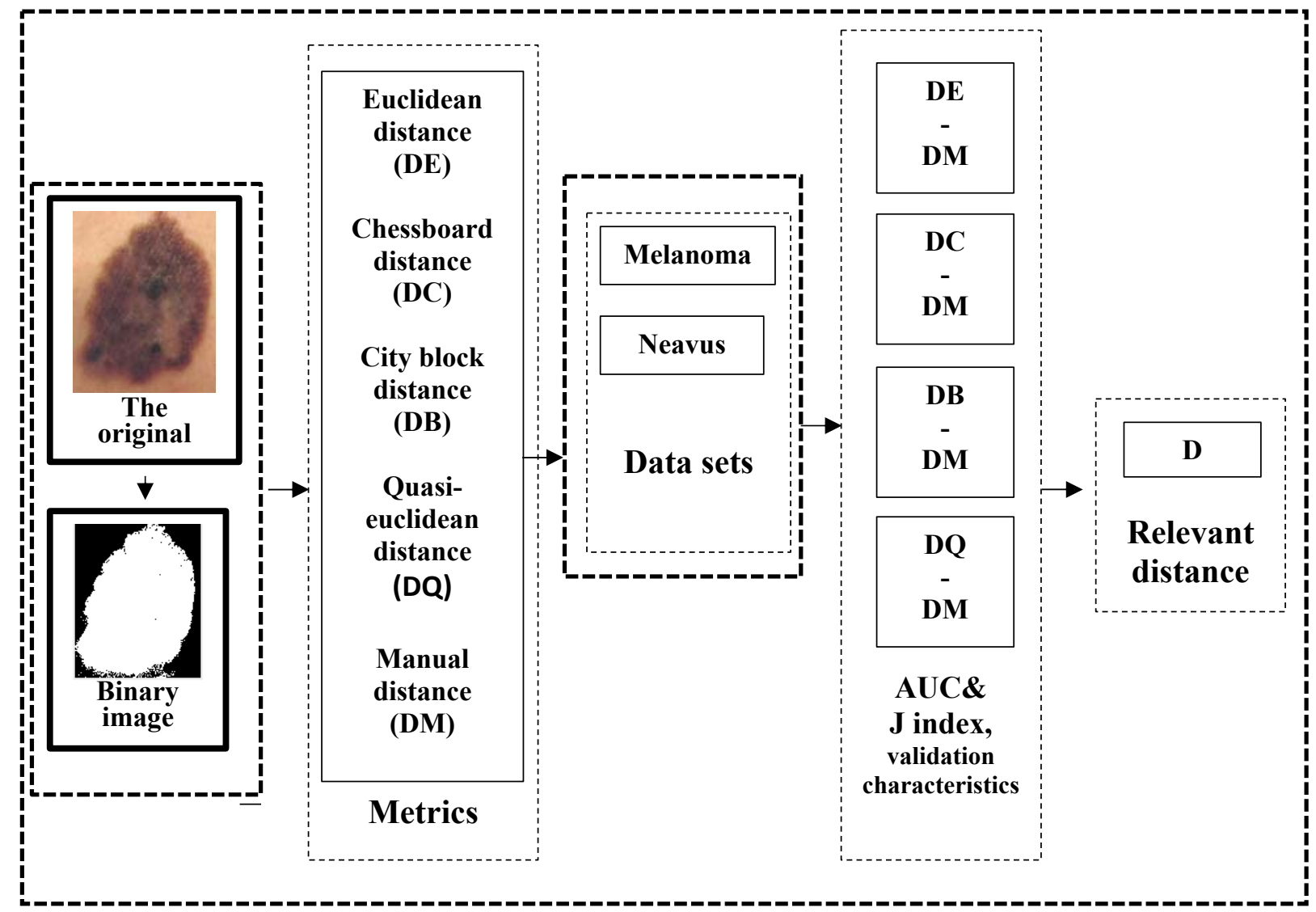

Fig. 1. Flowchart of the proposed method showing the steps involved in the processing of lesion images

The manually measured distance (MD) is determined using the Open Image Viewer app in the Matlab environment. First, the image is binarized, and then the maximum distance between two white pixels placed on the lesion boundary is determined. MD is the ground-truth for our method.

\section{RESULTS AND DISCUSSIONS}

A dataset containing 140 images (70 melanoma and 70 nevi) is investigated by using five distance metrics. The performance of the proposed metrics in differentiating between nevi and melanoma has been investigated using the area under the curve (AUC) and the Youden index derived from ROC curves.

The ROC curves, AUC values, and threshold values that were associated with the best Youden indices are presented in figures 3 and 4.

The stability of the proposed metrics is determined using the following threshold values: AUC $>0.6$ and the Youden index $\mathrm{J} \rightarrow 1$. 


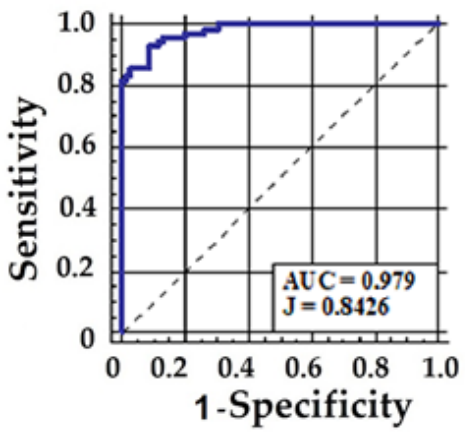

(a)

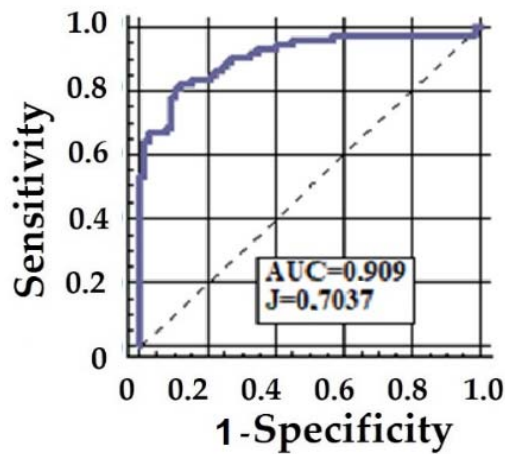

(c)

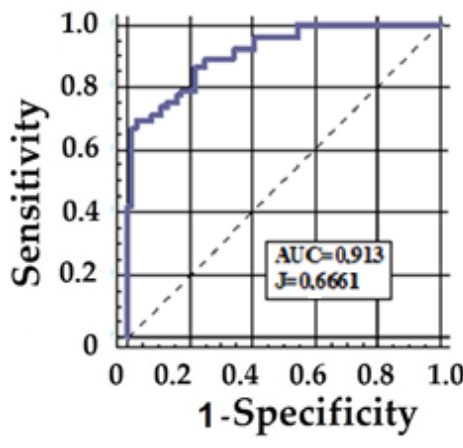

(b)

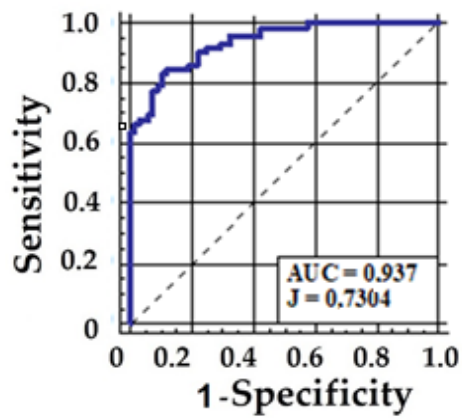

(d)

Fig. 3. Receiver Operating Characteristics ROC curves Area Under the Curve AUC and Youden index $J$ for discriminating distance metrics; (a) DC-DM, (b) DB-DM, (c) DE-DM and (d) DQ-DM, in the nevi case.

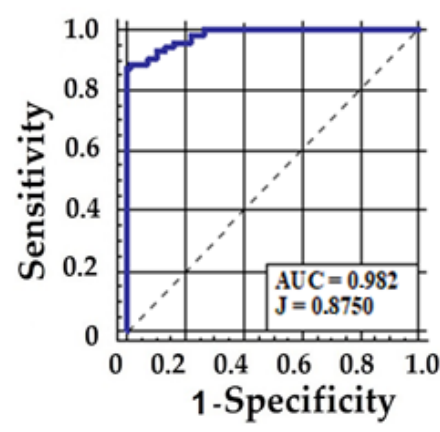

(a)

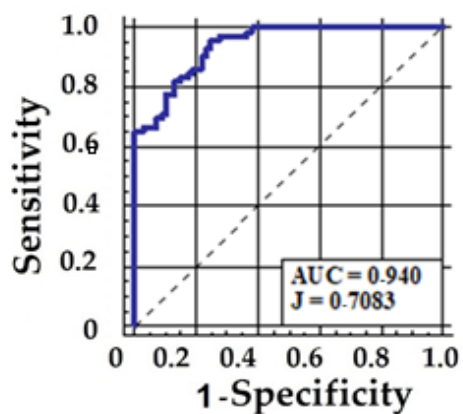

(c)

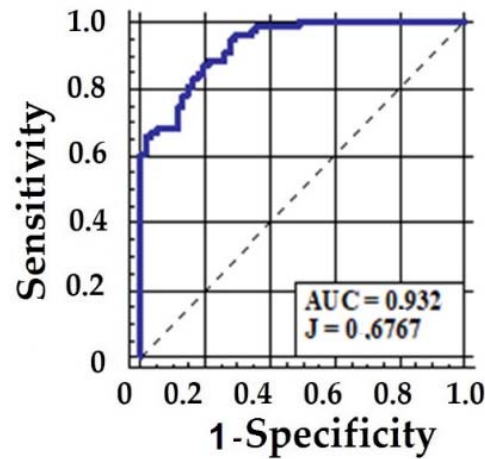

(b)

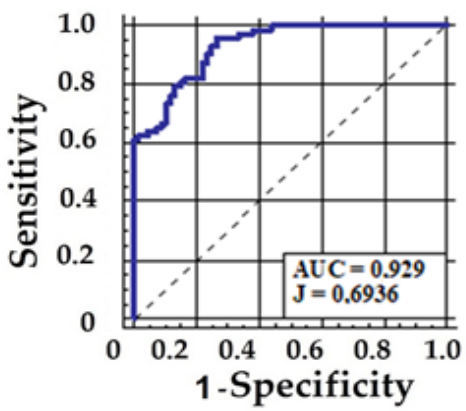

(d)

Fig. 4. Receiver Operating Characteristics ROC curves Area Under the Curve AUC and Youden index J for discriminating distance metrics; (a) DC-DM, (b) DB-DM, (c) DE-DM and (d) DQ -DM, in the melonoma case. 
All investigated distance metrics used for diameter measurement of the skin lesions had AUC values larger than 0.9. The best AUC values were determined for the Chessboard distance (DC) metric, namely 0.979 for nevi and 0.982 for melanoma. The Youden index $\mathrm{J}$ is 0.8426 for the nevi class, and 0.875 for the melanoma class indicating an excellent relevance of this metric for the declared goal. The smaller AUC values were obtained for the Euclidean distance (DE) with a value of 0.909 for the nevi class, and for the Quasi-Euclidean distance (DQ) with a value of 0.929 for the melanoma class, respectively. Similarly, the smaller values for J were obtained for the City block distance (DB) with 0.6661 for the nevi class and 0.6767 for the melanoma class, respectively.

The accuracy, sensitivity, and specificity are summarized in Table 1.

Table 1. The sensitivity, specificity, and accuracy of proposed distance metrics: DC-DM, DB-DM, DE-DM, and DQ- DM, in the nevi and melanoma case.

\begin{tabular}{|ccccc|}
\hline & DC-DM & DB-DM & DE-DM & DQ -DM \\
\hline $\begin{array}{c}\text { Sensitivity } \\
\text { (nevi) }\end{array}$ & 92.96 & 69.74 & 80.82 & 83.33 \\
$\begin{array}{c}\text { Sensitivity } \\
\text { (melanoma) }\end{array}$ & 87.50 & 87.34 & 95.83 & 92.83 \\
$\begin{array}{c}\text { Specificity } \\
\text { (nevi) }\end{array}$ & 91.30 & 96.87 & 89.55 & 89.71 \\
$\begin{array}{c}\text { Specificity } \\
\text { (melanoma) }\end{array}$ & 100.00 & 80.33 & 75.00 & 73.53 \\
$\begin{array}{c}\text { Accuracy } \\
\text { (nevi) }\end{array}$ & 92.13 & 83.30 & 85.18 & 86.52 \\
$\begin{array}{c}\text { Accuracy } \\
\text { (melanoma) }\end{array}$ & 93.75 & 83.83 & 85.41 & 84.68 \\
\hline
\end{tabular}

In terms of sensitivity and specificity, the Chessboard distance (DC) shows a balanced accuracy for nevi (92.13) and melanoma (93.75) (Table 1).

Conversely, city block (DB) has sensitivity values of 69.74/87.34 vs. specificity of 96.87/80.33 and 83.30/83.83 accuracy, respectively. Similarly, the values for Euclidean (DE) show sensitivity values of $80.82 / 95.83$ vs. specificity of $89.55 / 75.00$ and $85.18 / 85.41$ accuracy, and quasiEuclidean (DQ) metrics indicate a sensitivity of $83.33 / 92.83$ vs. specificity of $89.71 / 73.53$ and $86.52 / 84.68$ accuracy, respectively.

\section{CONCLUSIONS}

In this study, we have analyzed the capability of four distance metrics used to compute the diameter of skin lesions to differentiate between nevi and melanoma classes. The proposed method includes image binarization, lesion segmentation, diameter measurement, and classification-based on ROC, AUC, and J tools. The results show excellent performance rates for Chessboard distance, an AUC 0.979 for nevi and 0.982 for melanoma, respectively. Also, $\mathrm{J}$ is 0.8426 for nevi class, and 0.875 for melanoma class.

The study will be introduced in an app for differentiating common nevi from melanomas.

\section{References}

1. Damsky W. E, Bosenberg M., Melanocytic nevi and melanoma: unraveling a complex relationship, Oncogene 36 (42) (2017) 5771-5792.

2. Zaidi M. R., Fisher D. E., Rizos H., Biology of Melanocytes and Primary Melanoma, in: Cutaneous Melanoma, (Balch M.C., Atkins B.M., Garbe C., Gershenwald E.J., Halpern C.A., 
Kirkwood M.J., McArthur A.G., Thompson F.J., Sober J.A., editors), Springer, Cham, pp. 340, 2020.

3. Bi L., Kim J., Ahn E., Feng D., Fulham M., Automatic melanoma detection via multi-scale lesion-biased representation and joint reverse classification, IEEE $13^{\text {th }}$ International Symposium on Biomedical Imaging (ISBI), Prague, Czech Republic, (2016) 1055-1058.

4. Friedman R. J., Rigel D. S., Kopf A. W., Early detection of malignant melanoma: the role of physician examination and self-examination of the skin, CA: A Cancer Journal for Clinician, 35 (3) (1985) 130-151.

5. Goldsmith S. M., A unifying approach to the clinical diagnosis of melanoma including "D" for "Dark" in the ABCDE criteria, Dermatology Practical \& Conceptual, 4 (4) (2014) 75-78.

6. Daniel Jensen J., Elewski B. E., The ABCDEF Rule: Combining the "ABCDE Rule" and the "Ugly Duckling Sign" in an Effort to Improve Patient Self-Screening Examinations, Journal of Clinical and Aesthetic Dermatology 8 (2) (2015) 15.

7. Almaraz-Damian J.A., Ponomaryov V., Rendon-Gonzalez E., Melanoma CADe based on ABCD Rule and Haralick Texture Features, IEEE Conference Publication: $9^{\text {th }}$ International Kharkiv Symposium on Physics and Engineering of Microwaves, Millimeter and Submillimeter Waves (MSMW), Kharkiv, Ukraine, I (2016) 1-4.

8. Grammatikopoulos G., Hatzigaidas A., Papastergiou A., Lazaridis P., Zaharis Z., Kampitaki D., Tryfon G., Simple Matlab Tool for Automated Malignant Melanoma Diagnosis, WSEAS Trans. Inf. Sci. Appl., 4(3) (2007) 460-465.

9. Garnavi R., Aldeen M., Bailey J., Computer-aided diagnosis of melanoma using border and wavelet-based texture analysis, IEEE Transactions on Information Technology in Biomedicine, 16 (6) (2012) 1239-1252.

10. Kalwa U., Legner C., Kong T., Pandey S., Skin Cancer Diagnostics with an All-Inclusive Smartphone Application, Symmetry, 11 (6) (2019) 790.

11. Glazer A.M., Farberg A.S., Winkelmann R.R., Tucker N., Rigel D.S., Quantitative ABCD parameters measured by a multispectral digital skin lesion analysis device for evaluation of suspicious pigmented skin lesions strongly correlate with clinical ABCD observations, Dermatology Online Journal, 23(8) (2017) 1- 4.

12. Emiroglu N., Pelin Cengiz F., Hofmann-Wellenhof R., Dermoscopic and clinical features of trunk melanomas, Advances in Dermatology and Allergology, 31(6) (2014) 362-367.

13. Damian F.A., Moldovanu S., Dey N., Ashour A.S., Moraru L., Feature Selection of NonDermoscopic Skin Lesion Images for Nevus and Melanoma Classification, Computation, 8(2) (2020) 41 .

14. Hassan D., Aickelin U., Wagner C., Comparison of Distance metrics for hierarchical data in medical databases, International Joint Conference on Neural Networks (IJCNN), Beijing, China, (2014) 3636-3643.

15. Pandit S., Gupta S., A Comparative Study on Distance Measuring Approaches for Clustering, International Journal of Research in Computer Science, 2 (2011) 29.

16. Kamalov F., Leung H.-H., Chessboard metric and median spaces, Pure Math. Sci., 4 (2015) 133-137.

17. Prabhakar S. K., Rajaguru H., Code Converters with City Block Distance Measures for Classifying Epilepsy from EEG Signals, Procedia Comput. Sci., 87 (2016) 5-11.

18. Dong Hui, Han Dian Yuan, Research of image matching algorithm based on SURF features, International Conference on Computer Science and Information Processing (CSIP), Xi'an, Shaanxi, China, 1140-1143, 2012.

19. Westfall P. H., Kurtosis as Peakedness, 1905-2014. R.I.P., The American Statistician, 68(3) (2014) 191-195.

20. Damian F.A., Moldovanu S., Moraru L., Skin lesions analysis based on significant features selection algorithm, Proceedings of $8^{\text {th }}$ International Conference on "Experiments/ Process/ System Modeling/ Simulation/ Optimization”, Athens, Greece, II (2019) 380-387. 
21. James G., Witten D., Hastie T., Tibshirani R., Classification, in: An Introduction to Statistical Learning: with Applications in R, 2nd ed., (Casella G., Fienberg S., Olkin I., editors), Springer, New York, USA, pp. 147-149, 2013.

22. Ruopp M. D., Perkins N. J., Whitcomb B. W., Schisterman E. F., Youden Index and Optimal Cut-Point Estimated from Observations Affected by a Lower Limit of Detection, Biom. J., 50(3) (2008) 419-430.

23. Faraggi D., Simon R., A Simulation Study of Cross-Validation for Selecting an Optimal Cutpoint in Univariate Survival Analysis, Statistics in Medicine 15(20) (1996) 2203-2213. 